\title{
HIV INFECTION PRESENTING AS APLASTIC ANEMIA: A CASE REPORT
}

Fayaz Ahmad Wani' ${ }^{1}$, Lateef Ahmad Charoo ${ }^{2}$, Javid Rasool ${ }^{3}$, Roohi Rasool ${ }^{4}$.

1. Consultant Postgraduate, Department of Medicine, GMC Srinagar.

2. SMO ART Centre, Department of Medicine, Srinagar.

3. Consultant Department of Hematology, SKIMS Srinagar.

4. Consultant Department of Immunology, SKIMS Srinagar.

\section{CORRESPONDING AUTHOR: \\ Dr Fayaz Ahmad Wani, Consultant Dept of Medicine, GMC Srinagar. \\ Email- wanifayaz.a@gmail.com}

ABSTRACT: Disorders of the hematopoietic system including lymphadenopathy, anemia, leukopenia, and/or thrombocytopenia are common throughout the course of human immunodeficiency virus (HIV) infection and may be the direct result of HIV infection, manifestations of opportunistic infections and neoplasms, or side effects of therapy. However aplastic anemia due to HIV infection is very rare. Though anemia is seen with advanced disease and associated with poor prognosis, it is treated with various therapeutic modalities. Our patient with HIV infection had bone marrow documented aplastic anemia and responded very well to antiretroviral therapy.

KEY WORDS: Aplastic anemia; HIV; AIDS; Antiretroviral therapy.

INTRODUCTION: Hematological complications are a common cause of mortality in human immunodeficiency virus (HIV) infected patients. Cytopenias are most frequent during the advanced stage of disease. [1] The hematologic manifestations are multifactorial in etiology like direct effect of HIV, manifestation of opportunistic infection and neoplasm or side effect of therapy and the response to therapy depends upon the underlying etiological factor. [2] The prevalence of anemia in patients with acquired immunodeficiency syndrome (AIDS) has been estimated to be 63-95\%. [3] Treatment of HIV related anemia requires correction of nutritional deficiencies, blood transfusion, recmbinant human erythropoitin and modification of offending medication. ${ }^{[4]}$ We are reporting a case of aplastic anemia in a young girl secondary to HIV infection who responded very amenably to antiretroviral therapy (ART).

CASE REPORT: A 22 year old female developed skin rash, of one week duration for which she went to a dermatologist, who advised complete hemogram which revealed pancytopenia. Subsequently she developed bleeding gums and epistaxis, and was referred to clinical hematology department of our hospital in July 2008. There was history of weight loss. There was no history of fever, diarrhea, cough, anorexia. Two months back patient had mild jaundice which recovered without any treatment. There was a negative history of prior sexual contact. She had not received any blood transfusion. However she had received many injections from multiple practitioners for fatigue and aches and pains in the past. On examination she was ill looking, pale. She had purpuric spots all 
over the body. She had significant weight loss (10\%). There was no lymphadenopathy, hepatomegaly or splenomegaly. Rest of the systemic examination was normal. Her hemogram showed hemoglobin of 8.7 gms\%, with total leucocyte count of 1,200 cell/ cumm and platelet count was 5,000 cells/ cumm. Her absolute neutrophil count was 242 cells/ cumm. Her reticulocyte count was $0.5 \%$. Her serum chemistry was within normal limits [Table 1]. Bone marrow aspiration and biopsy were done [Figures 1-3] which revealed grossly hypo cellular marrow with little cellular fragments. Smear showed mainly lymphocytes, RE cells and few erythroid cells. No megakaryocytic cells were seen. There were no parasites, excess blasts or myxomatous changes. Iron stain was 3+. All these findings were suggestive of aplastic anemia. She was investigated for secondary causes of aplastic anemia, HIV serology done in august 2008 showed Accu Check rapid diagnostic test, Insta Check HIV 1+2 triline test and HIV MICROLISA (ELISA) test all reactive. HIV status was confirmed by western blot. Her CD4 count was 33 cells/ $\mu$ land CD4 percent of 15 . ANA was negative; CMV and EBV serology was negative. Vitamin B12 and folate levels were within normal limits. She was labeled as a case of aplastic anemia secondary to HIV infection. Both the parents were screened for HIV but were found to be negative. In view of bone marrow suppression due to HIV infection and low CD4 count, she was started on antiretroviral therapy in august 2008 in the form of Stavudine (d4T), Lamivudine (3TC) and Efavirenz. Zidovudine was avoided in view of anemia. She was followed up regularly with clinical and hematological examination. After ten months on antiretroviral therapy, she has improved symptomatically with no bleeding at present. Her appetite improved and weight has increased by 8 kilograms. Her hemogram has shown remarkable improvement [Table 2]. A repeat bone marrow examination was contemplated, but patient refused this invasive procedure as she is doing well on treatment. She has tolerated antiretroviral therapy well, without any major side effects. However she could not tolerate cotrimoxazole (prophylaxis) due to gastric irritation.

DISCUSSION: Hematological abnormalities are among the most common complications of HIV. These involve all lineages of blood cells. [1] HIV associated hematological abnormalities seem to be dependent on the level of virus replication, as these abnormalities are severe in late-stage AIDS patients with high viremia. A specific diagnosis of the cause and mechanism must be sought because specific treatment may be needed for its correction. [1] In recent years several attempts have been undertaken to elucidate the mechanisms leading to HIV-associated anemia.

Opportunistic complications and myelophthisis caused by malignancies such as lymphoma represent the underlying cause for anemia in a large number of HIV-infected patients. Next to this rather obvious reason for anemia, iatrogenic anemia induced by myelosuppressive drugs is also very common. [5] Deficiency of vitamin B12, folate and iron is frequently reported in HIV patients. Other studies have suggested that soluble factors in the serum of HIV-infected patients may inhibit haematopoiesis, or that direct HIV infection of marrow progenitor cells may play a role in producing anaemia and other hematologic abnormalities associated with HIV infection. [6]

A significant percentage of bone marrow aspirates from patients with HIV infection have been reported to contain lymphoid aggregates, the precise significance of which is unknown. Bone marrow abnormalities are found in all stages of HIV disease, increasing in frequency as the disease progresses. A number of characteristic but nonspecific, morphologic abnormalities of the bone marrow of AIDS patients have been reported. [7] Aplastic anemia due to direct HIV infection of 
marrow progenitor cells has been rarely reported in literature with only two cases reported including one from India. [8]

In the present patient the bone marrow examination was not suggestive of any opportunistic infection or malignancy, serology for opportunistic infection was negative, patient was not on any drugs before presentation and vitamin B12, folate and iron levels were normal, so all these factors as a cause of anemia in this patient were excluded. Since the patient improved very well on ART at the end of 10 months, the most likely cause of bone marrow suppression in this patient is direct HIV infection of marrow progenitor cells.

Treatment of hematological manifestations of HIV addresses the underlying cause such as treatment of opportunistic infections and malignancies, modification of offending medication and correction of nutritional deficiencies. For anemia that is not amenable to correction, treatment by blood transfusion and recombinant human erythropoietin are recommended. [4] Bone marrow transplant is not very useful in aplastic anemia with HIV. [9] In the present patient of aplastic anemia due to HIV infection, we saw an excellent clinical and hematological response to combined ART. Thus we suggest that every aplastic anemia patient should be screened for HIV infection, as HIV infection itself may lead to bone marrow suppression and respond to ART only.

\section{REFERENCES:}

1. Kirchhoff F, Silvestri G. Is Nef the elusive cause of HIV-associated hematopoietic dysfunction? J. Clin. Invest 2008, 118:1622-5.

2. Kasthuri AS, Sharma Sanjeevan, Kar PK. A study of haematological manifestations of HIV Infection. Indian J Sex Transm Dis 2006; 27(1): 9-16.

3. Mir N, Castelo C, Luckit J, Lindley R. HIV disease and bone marrow changes: A study 60 cases. Eur J Haematol 1989: 42: 339-340.

4. Claster S. Biology of anemia, differential diagnosis and treatment options in human immunodeficiency virus infection. J Infect Dis 2002; 185 (suppl.2): 105-109.

5. Doukas MA. Human deficiency virus associated anemia. Med Clin North AM 1992; 76: 699700.

6. Folks TM, Kessler SW, Orenstein JM, et al. Infection and replication of HIV-1 in purified progenitor cells of normal human bone marrow. Science 1988;242:919-922

7. Frontiera M, Myers AM. Peripheral blood and bone marrow abnormalities in the acquired immunodeficiency syndrome. West J Med 1987, 147:157-60.

8. Ira Shah, Anuradha K Murthi. Aplastic anemia in an HIV infected child. Indian J Pediatr 2005; 72 (4): 359-361.

9. Giri N, Vowels MR, Ziegler JB. Failure of allogenic bone marrow transplantation to benefit HIV infection. J Paediatr Child Health 1992; 28: 331-333. 
TABLE 1: Serum bio-chemistry of the patient.

\begin{tabular}{|l|l|l|l|}
\hline Date & $31 / 7 / 08$ & $02 / 8 / 08$ & $12 / 8 / 08$ \\
\hline Blood glucose(R) (mg\%) & 86 & 73 & 118 \\
& & & \\
\hline Urea(mg\%) & 21 & 22 & 25 \\
\hline Creatinine(mg\%) & 0.5 & 0.6 & 0.7 \\
\hline Bilirubin(mg\%) & 1.10 & 1.10 & 0.88 \\
\hline SGOT (U/L) & 27 & 51 & 37 \\
\hline SGPT (U/L) & 45 & 47 & 50 \\
\hline ALP (U/L) & 223 & 246 & 186 \\
\hline Total Protein (mg\%) & 7.02 & 8.22 & 7.94 \\
\hline Albumin(mg\%) & & & \\
\hline LDH & 4.2 & 4.4 & 5.2 \\
\hline
\end{tabular}

TABLE 2: Follow-up hemogram of the patients.

\begin{tabular}{|l|l|l|l|l|l|l|l|l|}
\hline & $2 / 8 / 08$ & $12 / 8 / 08$ & $3 / 9 / 08$ & $27 / 11 / 08$ & $12 / 1 / 09$ & $5 / 3 / 09$ & $3 / 4 / 09$ & $16 / 6 / 09$ \\
\hline $\mathrm{Hb}$ & 7.90 & 6.9 & 5.8 & 4.3 & 6.2 & 10.2 & 10.8 & 11.8 \\
\hline TLC & 0.66 & 0.33 & 1.08 & 1.50 & 2.1 & 2.62 & 2.78 & 4.01 \\
\hline $\mathrm{N}$ & 0.01 & 0.12 & 0.28 & 0.60 & 0.95 & 1.14 & 1.05 & 1.88 \\
\hline $\mathrm{L}$ & 0.9 & 0.78 & 0.57 & 0.77 & 1.0 & 1.20 & 1.38 & 1.72 \\
\hline Plt & 11 & 3 & 5 & 12 & 25 & 33 & 46 & 83 \\
\hline CD4 & & 33 & & & & 132 & & \\
\hline
\end{tabular}




\section{CASE REPORT}

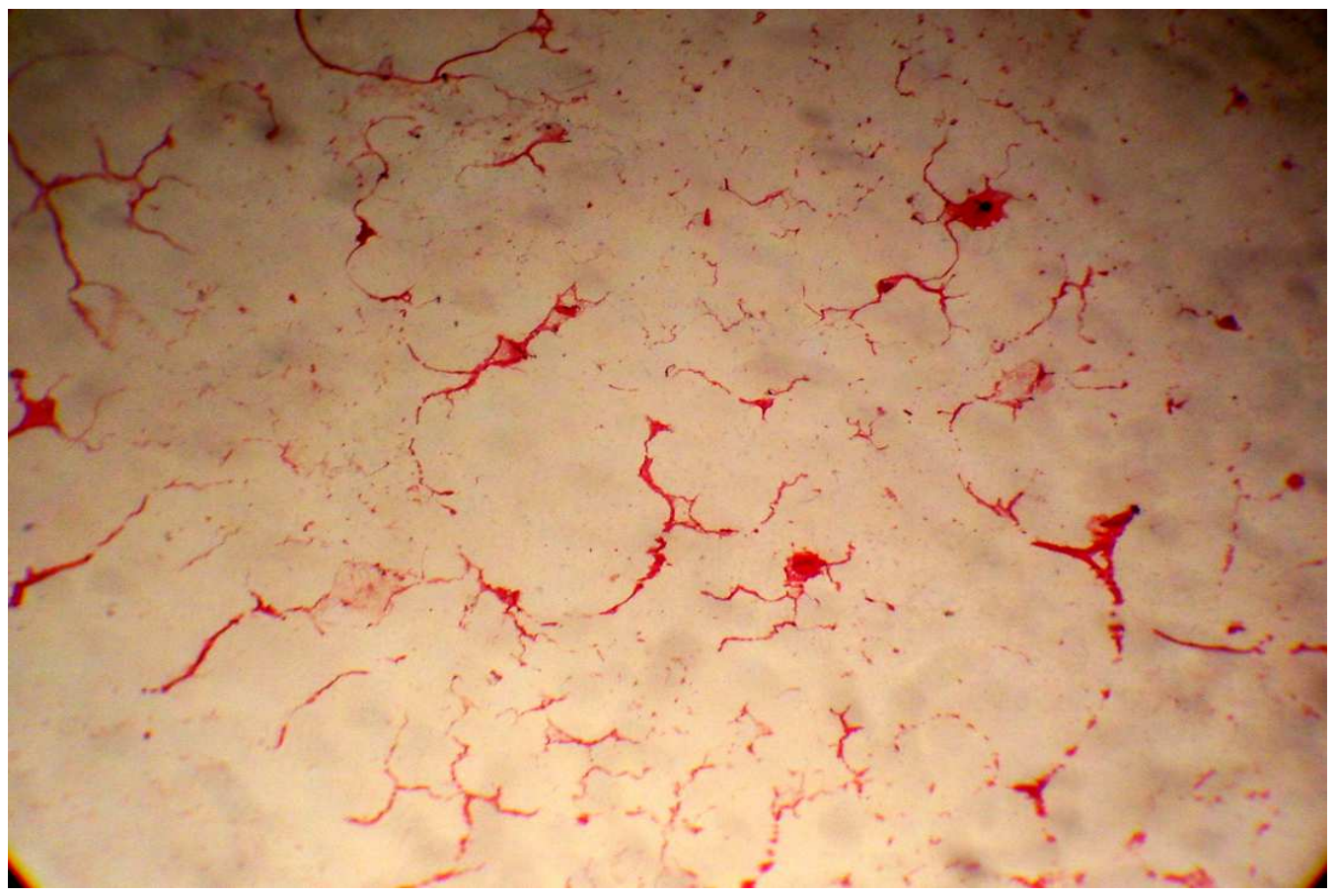

LEGEND TO FIGURE 1: Bone marrow trephine showing absence of hematopoietic tissue and its replacement by fat.

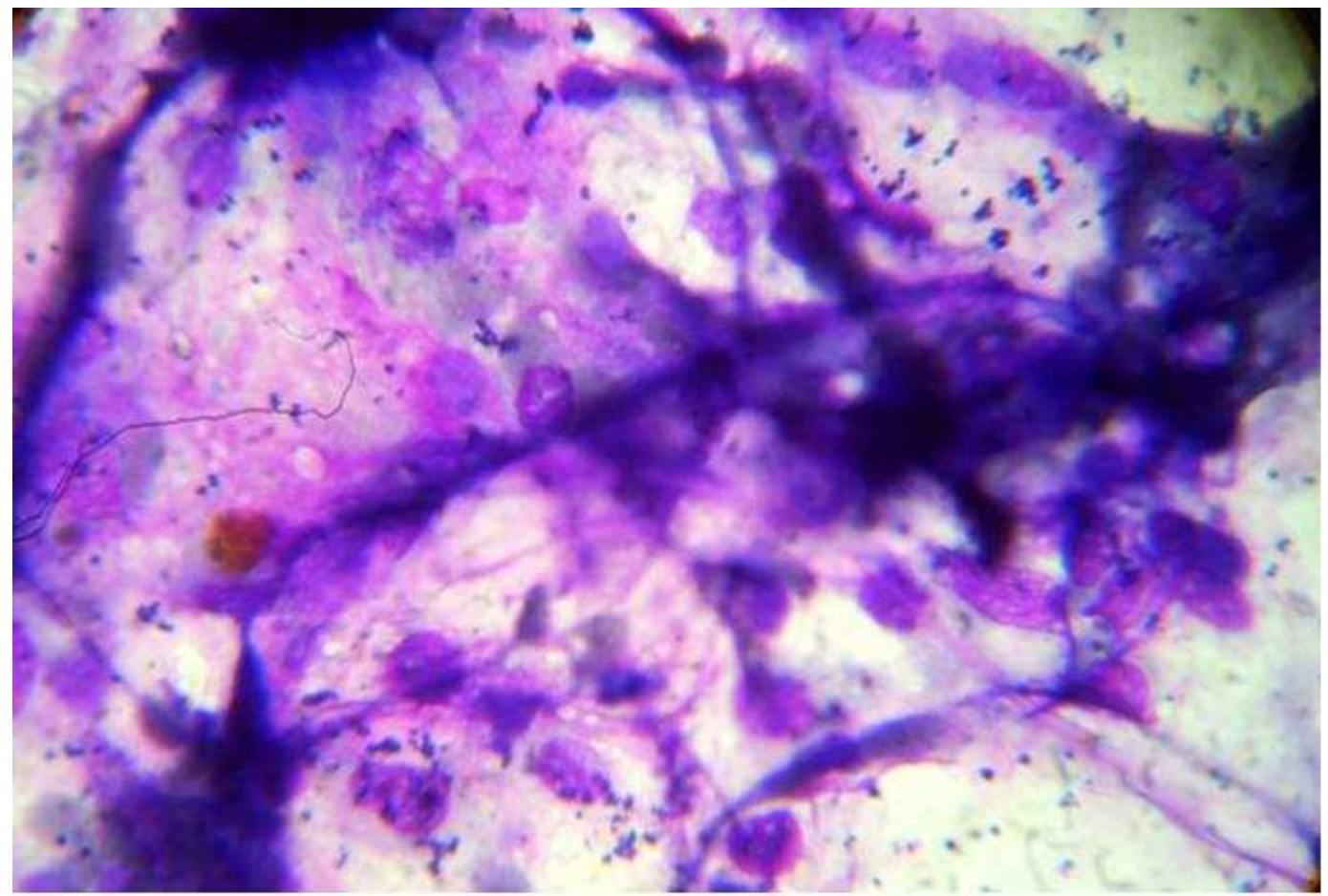

LEGEND TO FIGURE 2: Bone Marrow aspiration showing grossly hypocellular fragment with RE meshwork. 


\section{CASE REPORT}

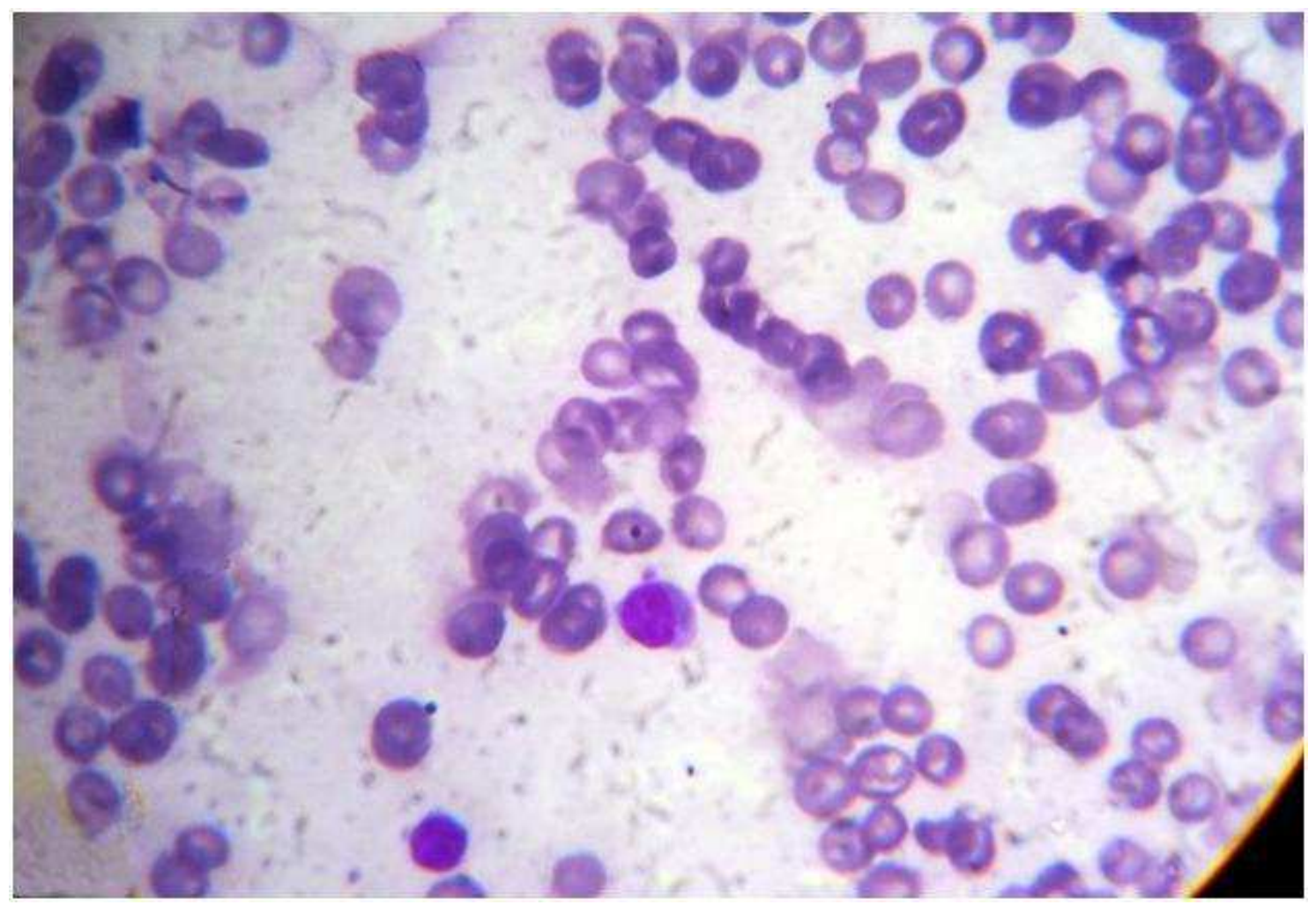

LEGEND TO FIGURE 3: Bone Marrow aspiration smears showing grossly hypocellular cell trails 